\title{
Saddle-point construction in the design of lithographic objectives, part 2: application
}

\author{
Oana Marinescu \\ Florian Bociort, MEMBER SPIE \\ Delft University of Technology \\ Optics Research Group \\ Lorentzweg 1 \\ 2628 CJ, Delft \\ The Netherlands \\ E-mail: o.marinescu@gmail.com
}

\begin{abstract}
Optical designers often insert or split lenses in existing designs. Here, we apply in the design of objectives for deep and extreme UV lithography an alternative method for adding new components that consists of constructing saddle points in the optical merit function landscape and obtaining new local minima from them. The design examples show that this remarkably simple method can be easily integrated with traditional design techniques. The new method has significantly improved our design productivity in all cases in which we have applied it so far. High-quality designs of lithographic objectives are obtained with this method. () 2008 Society of Photo-Optical Instrumentation Engineers. [DOI: 10.1117/1.2981513]
\end{abstract}

Subject terms: saddle point; lithography; optimization; optical system design; EUV; DUV.

Paper 080163R received Mar. 4, 2008; revised manuscript received Jul. 24, 2008; accepted for publication Jul. 25, 2008; published online Sep. 30, 2008. This paper is a revision of a paper presented at the SPIE conference on Optical Design and Engineering II, September 2005, Jena, Germany. The paper presented there appears (unrefereed) in SPIE Proceedings Vol. 5962.

\section{Introduction}

In the traditional design process of optical systems, lenses are frequently inserted or split in existing configurations. An alternative way to achieve the same goals, which is based on constructing saddle points in the optical merit function landscape, has been presented recently. ${ }^{1}$

In the first part of the present study, the saddle-point construction method was discussed in detail and its applicability to deep UV (DUV) and extreme UV (EUV) lithography was shown. ${ }^{2}$ The central idea of the method is that, at any position in a local minimum with $N$ surfaces, inserting a thin meniscus lens (or two mirrors) in contact with an existing surface leads to a system with $N+2$ surfaces that is a saddle point. Any optical merit function can be used, e.g., one based on transverse aberrations (root-mean-square spot size), wavefront aberration, etc.

We start from an optimized system that has $N$ surfaces. At an arbitrary position in the system, we insert a meniscus lens with zero thickness and equal curvatures in contact with (i.e., at zero axial distance from) an existing surface (the reference surface). The meniscus should be made of the same material as the lens with the reference surface. In this special case, the curvatures of the meniscus lens should be equal to the curvature of the reference surface. ${ }^{1,2}$ In this way, where we insert the thin meniscus we have three surfaces with equal curvatures and zero axial distances between them. We select two points situated on opposite sides of the saddle by slightly modifying two consecutive curvatures in the resulting system (saddle point). Via local optimization performed starting from these points, two new local minima, with $N+2$ surfaces, are then generated. Finally, at each minimum we increase the thickness of the

0091-3286/2008/\$25.00 @ 2008 SPIE inserted meniscus lens and the distance between it and the surface where it was introduced. The method is described in detail in the accompanying paper ${ }^{2}$ (see also Ref. 3).

Generating optical systems having new shapes, by using the saddle-point construction method, can be done in a very short time in comparison with the traditional design techniques. In general, the time necessary to create a saddle point and obtain two local minima from it (by means of local optimization), as just described, is of the order of a few minutes. Increasing the axial distance between the surfaces is related to the local optimization process (merit function construction), and it depends on the complexity of the optical system.

Despite the fact that it is based on nontrivial new insight into the properties of the optical design space, the method is remarkably simple and computationally efficient and can be applied to the design of systems of arbitrary complexity. When a lens is inserted so that a saddle point is constructed, two local minima are generated after optimization, whereas by inserting a lens in the traditional way, we always obtain a single local minimum. With the new method, the better of the two minima can then be used for further design.

In this second part of the study, we present design results obtained with the new method. We first apply the saddlepoint construction method to the design of DUV lithographic objectives and show that, even for excellent designs, there can still be potential for further improvement. Then, we show how the method can be used to generate EUV mirror designs having new shapes. All results presented in this article have been obtained using the optical design program CODE V.

\section{Dioptric Deep UV Lithographic Objective}

In this section, we show how by first adding lenses via constructed saddle points and then removing lenses from 


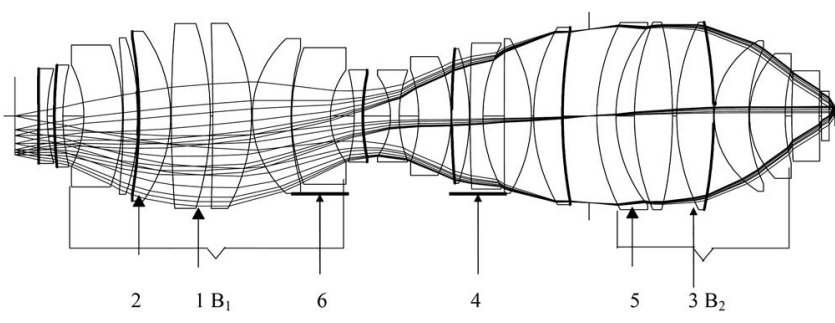

Fig. 1 The starting design is a 0.85 NA lithographic objective for $193 \mathrm{~nm}$. Surfaces drawn with a thicker line are aspherical.

the local minimum with the better imaging performance, obtained from the saddle point, we can obtain a lithographic objective with a reduced number of lenses, but with performance that is not worse than that of the initial system.

In the examples discussed in this paper, all lenses are made of the same material. Figure 1 shows our starting system: a lithographic objective for $193 \mathrm{~nm}$, which has already high imaging quality. ${ }^{4}$ The system has a numerical aperture of 0.85 , the image height is $14.02 \mathrm{~mm}$, and the magnification is -0.25 . The distortion is below $4.2 \mathrm{~nm}$ per field point, the Strehl ratio is 0.999 , and the root mean square (rms) wavefront aberration is $3.67 \mathrm{~m} \lambda$.

We use a merit function based on rms wavefront aberration. Distortion and telecentricity on the object and image sides are also controlled. The first bulge, $\mathrm{B}_{1}$, consists of seven lenses, having spherical and aspherical surfaces. All lens thicknesses have been made equal, as well as the distances between them. In contact with the surface indicated by arrow 1, a zero-thickness meniscus lens has been inserted to construct a saddle point. On letting the optimization roll down on the two sides of the saddle (for details see Refs. 2 and 5, two local minima are generated. In each local minimum the thickness of the thin meniscus and the distance between it and the reference surface have been gradually increased to the same values as those of the other lenses in $\mathrm{B}_{1}$. We have continued the design with the solution having the better performance of the two, in terms of rms wavefront aberration. In this system it was then possible to extract the lens indicated with arrow 2 (which has an aspheric surface), as well as the spherical lens that has just been inserted. The resulting minimum (Fig. 2) has two surfaces less than the starting system shown in Fig. 1. Moreover, it has one aspheric surface (described by seven aspheric coefficients) less. The rms wavefront aberration is $4.57 \mathrm{~m} \lambda$, slightly larger than that of the starting system, but

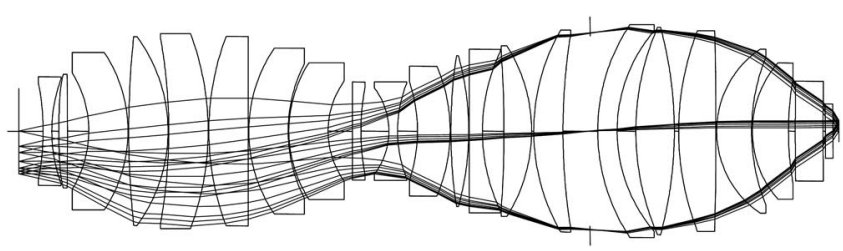

Fig. 2 The 0.85 NA lithographic objective after extracting from the first bulge a lens with an aspheric surface.

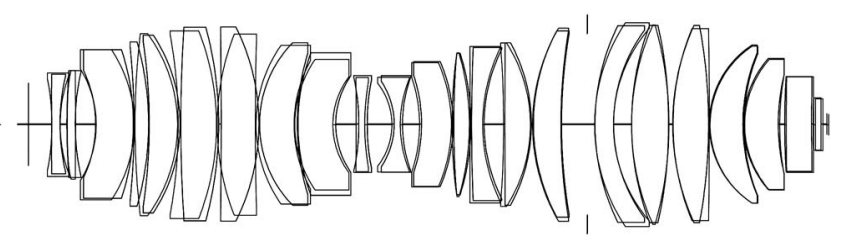

Fig. 3 Comparison between the starting system (Fig. 1) and the configuration shown in Fig. 2. For facilitating comparison, the two system drawings have been superimposed.

this is also due to the fact that at this stage the thicknesses of the lenses in this bulge do not yet have the optimal value.

To extract lenses, we have used the same strategy as that discussed in the accompanying article. ${ }^{2}$ The thickness of the lens to be removed and the distance between the lens and the preceding or following one are reduced in several steps to zero. The shapes of the surfaces of the resulting thin lens are then made identical to that of the surface with which they are in contact. Then, the thin meniscus thus obtained can be removed without affecting the system performance. $^{6}$

Comparing the two configurations, we observe that the most significant differences appear around the region where we have inserted the meniscus and extracted the lens (Fig. 3). As observed in other cases as well, ${ }^{2}$ the rest of the lenses remain almost unchanged. These lenses can be frozen during the process of extracting lenses and released at the final stage to improve the resulting system. This also supports the conjecture according to which changing a limited number of relevant variables is sufficient to place the local optimization in the basin of attraction of the solution that is a local minimum for all variables.

Next, a thin meniscus is inserted in the second bulge of the system, $B_{2}$ (see arrow 3 in Fig. 1). In the better local minimum connected to the constructed saddle point, we have merged the two lenses indicated with arrow 4. The resulting configuration allowed us to extract lens 5 , as well as to merge the two lenses shown in Fig. 1 with arrow 6. Finally, the system has been optimized with respect to all variables (curvatures, aspheric coefficients, and distances).

The resulting design in Fig. 4 has three lenses and one aspheric surface less than the starting system shown in Fig. 1. Despite this fact, its performance, in terms of rms wavefront aberration, distortion, telecentricity, and Strehl ratio, is actually slightly better than that of the starting system. The rms wavefront aberration is $2.37 \mathrm{~m} \lambda$, lower than that of the starting system. The Strehl ratio is 0.9998 , which can

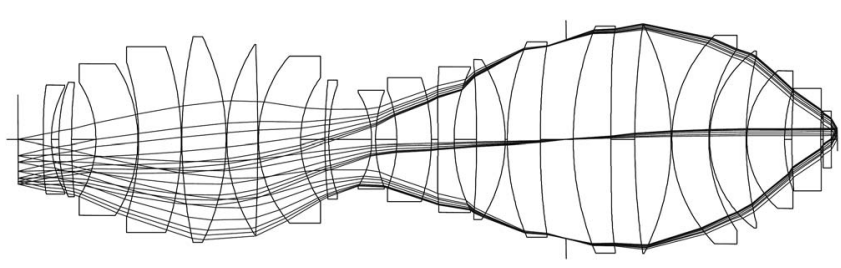

Fig. 4 Design with a numerical aperture of 0.85 , an image height of $14 \mathrm{~mm}$, and a rms wavefront aberration of $\mathrm{m} \lambda$, obtained after extracting three lenses and an asphere from the design shown in Fig. 1. 
Table 1 Specifications and performance for the DUV lithographic objective shown in Fig. 4.

\begin{tabular}{lc}
\hline \hline Specification & Value \\
\hline $\mathrm{NA}$ & 0.85 \\
Image field & $14.02 \mathrm{~mm}$ \\
Wavelength & $193.368 \mathrm{~nm}$ \\
Magnification & -0.25 \\
Distortion & $<1 \mathrm{~nm}$ \\
Strehl ratio & 1.000 \\
Rms wavefront aberration & $2.37 \mathrm{m \lambda}$ \\
\hline \hline
\end{tabular}

be taken as unity for all practical purposes. The distortion is smaller than $1 \mathrm{~nm}$, and the telecentricity is slightly better than in the original design. A summary of the system specifications and performance is shown in Table 1, and detailed description of the configuration, including the optical prescription can be found in Ref. 6. Such a reduction in the number of elements without image quality degradation can lead to an appreciable decrease in the fabrication cost of an optical system.

\section{Catadioptric Configuration}

The use of the saddle-point construction method for obtaining new system shapes is illustrated in this section on a catadioptric configuration designed for DUV lithography at $157 \mathrm{~nm}$. The starting system (see Fig. 5) comprises 27 optical elements.

A thin meniscus lens was inserted in the second bulge of the system (comprising lenses 5 to 9) so that a saddle point was constructed. From the solution with the better imaging performance obtained from the saddle point after the thickness of the thin meniscus has been increased, a lens was removed from the second bulge of the system. Then a lens was extracted from the last bulge of the system, and finally the second lens of the initial system was removed. The numerical aperture was then increased from 0.845 to 0.88 . For object fields smaller than $40 \mathrm{~mm}$, the rms wavefront aberration is smaller than $7.8 \mathrm{~m} \lambda$. Further improvement might still be possible, but this design stage (see Fig. 6) shows already the potential of the method to create new system shapes for very complex systems.

Considering the total amount of effort required for designing a DUV or EUV objective, we have observed in all

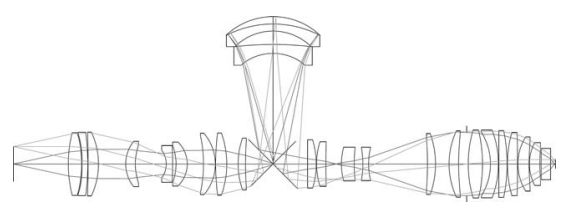

Fig. 5 Starting catadioptric configuration for $157 \mathrm{~nm}$ lithography. The numerical aperture is 0.845 , the image height is $13.6 \mathrm{~mm}$, and the magnification is 0.25 .

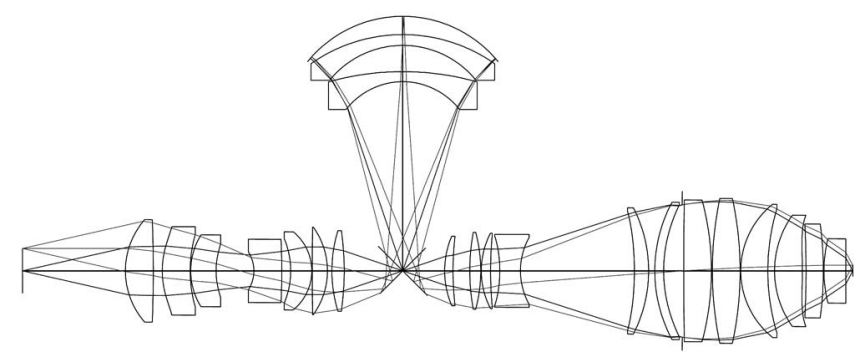

Fig. 6 Configuration with a rms wavefront aberration of $7.8 \mathrm{~m} \lambda$ and a distortion smaller than $1 \mathrm{~nm}$ obtained from that in Fig. 5, after extracting three lenses. The numerical aperture is 0.88 .

cases that changing the system shape with our method can be done in a time that is short in comparison with the time we have spent for further refining the new configuration with traditional techniques, including local optimization, to meet practical requirements.

The saddle-point construction method has also been successfully used in reshaping DUV lithographic objectives of a different type, having a larger numerical aperture than that of the systems so far presented here.

\section{Design of an Extreme UV Projection System}

Using the saddle-point construction method, we have obtained an eight-mirror design for EUV lithography from a six-mirror design with spherical surfaces. In the starting system $^{8}\left(m_{6}\right.$ in Fig. 7$)$ a pair of spherical mirrors has been inserted after the second surface. From the constructed saddle point, two solutions have been generated by means of local optimization, each having three consecutive mirrors in contact. After increasing the zero axial distances, the solutions have been locally optimized with respect to all variables (curvatures, aspheric coefficients, and distances) and practical constraints. For the two systems obtained in this way, the numerical aperture has been increased from 0.16 to 0.4 , and the systems have been reoptimized. The two resulting configurations, $m_{8,52 A}$ and $m_{8, S 2 B}$, are shown in Fig. 7. The performance of $m_{8, S 2 B}$ is poor. The solution $m_{8, S 2 A}$ satisfies practical requirements: distortion smaller

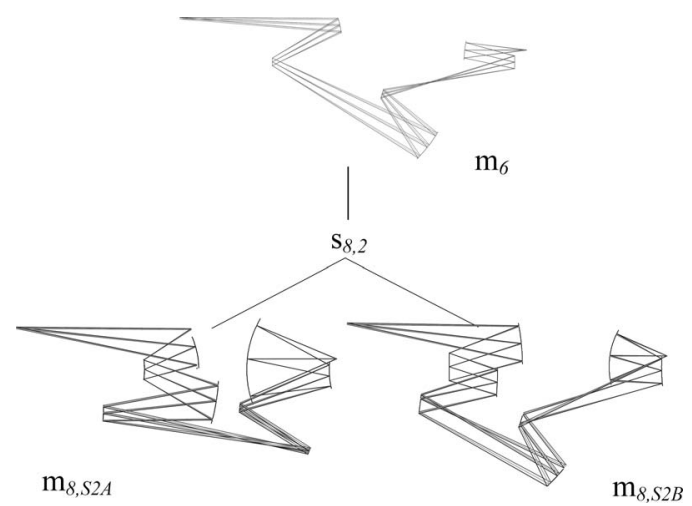

Fig. 7 A saddle point has been constructed from $m_{6}$ by inserting a pair of mirrors at the second surface. Solutions with eight surfaces resulting from a constructed saddle point, $s_{8,2}$, have been reoptimized for a numerical aperture of 0.4 . The local minimum $m_{8, S 2 B}$ is poor, but for $m_{8, S 2 A}$ the Strehl ratio is larger than 0.996 and the distortion is below $1 \mathrm{~nm}$. 
Table 2 Specifications and performance of the EUV mirror system $m_{8, S 2 A}$ in Fig. 7.

\begin{tabular}{lc}
\hline \hline Specifications & Value \\
\hline NA & 0.4 \\
Field & 114 to $118 \mathrm{~mm}$ \\
Wavelength & $13.4 \mathrm{~nm}$ \\
Magnification & 0.25 \\
Distortion & $<1 \mathrm{~nm}$ \\
Strehl ratio & $>0.996$ \\
Rms wavefront aberration & $10 \mathrm{m \lambda}$ \\
\hline \hline
\end{tabular}

than $1 \mathrm{~nm}$ for each field position, and Strehl ratio larger than 0.996 , with a rms wavefront aberration of $10 \mathrm{~m} \lambda$. All surfaces are aspheric and the system has an intermediate image between mirrors 4 and 5. The aperture stop is situated on the second mirror. Some of the system's specifications are shown in Table 2. (The entire specification list can be found in Ref. 6.) During optimization, a constraint was used to prevent obstruction, but in the last optimization cycles this constraint was inactive. The final design is unobstructed. At the wafer side the system is telecentric, i.e., the chief ray is (approximately) perpendicular on the image plane. For coating-related reasons, the angular spread at each surface has been kept smaller than $10 \mathrm{deg}$, as can be seen in Table 3.

\section{Conclusions}

The design of lithographic objectives and other complex optical systems is time-consuming. An advantage of the method discussed in this article is that it is straightforward, fast, and easy to use. In fact, for lithographic objectives, the

Table 3 Chief-ray angles of incidence and the variation of the angle of incidence (angular spread) at each surface of the EUV mirror system presented in Fig. 7. The angle of incidence of the chief ray at the mask is approximately 6.3 deg.

\begin{tabular}{lcc}
\hline \hline Mirror & $\begin{array}{c}\text { Angle } \\
(\mathrm{deg})\end{array}$ & $\begin{array}{c}\text { Angular spread } \\
(\mathrm{deg})\end{array}$ \\
\hline 1 & 15 & 0.26 \\
2 & 23.8 & 2.06 \\
3 & 15 & 1.01 \\
4 & 8.3 & 3.66 \\
5 & 10.75 & 1.16 \\
6 & 25.72 & 0.43 \\
7 & 15.63 & 9.18 \\
8 & 5.86 & 1.92 \\
\hline \hline
\end{tabular}

time required to change the shape of the configuration is typically small in comparison with the time required for further refinement of the new configuration with traditional design techniques. Inserting a lens in an optical system in the traditional way leads after local optimization to a single solution. When the insertion is performed so that a saddle point is created, two solutions can be obtained after optimization. Therefore, systems having new shapes are detected, which otherwise, using the traditional way, might not have been found. So far, we have applied the new method in several designs of DUV dioptric and catadioptric lithographic objectives, and of EUV objectives. In all these cases, it has significantly improved our design productivity.

\section{Acknowledgments}

The first author thanks ASML and Carl Zeiss SMT AG for the financial support of this research. The authors thank Willi Ulrich, Heiko Feldmann, and other members of the optical design team from Carl Zeiss SMT AG for valuable discussions.

\section{References}

1. F. Bociort and M. van Turnhout, "Generating saddle points in the merit function landscape of optical systems," Proc. SPIE 5962, 213220 (2005).

2. O. Marinescu, and F. Bociort, "Saddle-point construction in the design of lithographic objectives, part 1: method," Opt. Eng. 47(9), 093002 (2008) (this issue)

3. F. Bociort, M. van Turnhout, and O. Marinescu, "Practical guide to saddle-point construction in lens design," Proc. SPIE 6667, 666708 (2007). Lens data files for the examples given here are available at http://wwwoptica.tn.tudelft.nl/users/bociort/SPC_guide.zip

4. W. Ulrich, R. Hudyma, and H. J. Rostalski, "Refractive projection lens," German patent No. WO2003075096 (2003).

5. F. Bociort, E. van Driel, and A. Serebriakov, "Networks of loca minima in optical system optimization," Opt. Lett. 29(2), 189-191 (2004).

6. O. E. Marinescu, "Novel design methods for high-quality lithographic objectives," $\mathrm{Ph}$ D Thesis, Delft University of Technology, Sieca, http://wwwoptica.tn.tudelft.nl/publications/Thesis/ Marinescu.pdf

7. Y. Omura, "Projection optical system and exposure apparatus having the projection optical system," German patent No. WO/2003/036361 (2003).

8. M. F. Bal, F. Bociort, and J. J. M. Braat, "Lithographic apparatus, device manufacturing method and device manufactured thereby," U.S. Patent No. 6,556,648 (2003).

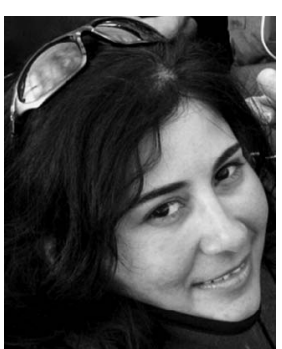

Oana Marinescu graduated from the Faculty of Physics at West University of Timisoara, Romania in 2001. In 2006, she received her $\mathrm{PhD}$ in physics from Delft University of Technology, The Netherlands. Her professional experience includes developing and testing design methods for extreme and deep UV lithography, designing lithographic objectives and illumination systems, tolerance analysis, and radiometric measurements. Currently, she is employed as an optical designer by TNO in Delft, The Netherlands.

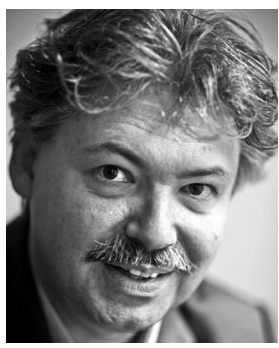

Florian Bociort is a lecturer with the Optics Research Group at Delft University of Technology, Delft, The Netherlands. His research interests include optimization, with special emphasis on the topology of highdimensional merit function landscapes, and the design of deep-UV and extreme-UV lithographic objectives. He holds a $\mathrm{PhD}$ in physics from the Technical University of Berlin, Germany. 